\title{
Space Charge Dynamics and Electric Field Distortion in the Laminated insulation for HVDC Cable
}

\author{
Zhiqiang Xu, Miao Hao, Bo Huang, George Chen, Matt Praeger and Paul Lewin \\ University of Southampton \\ Southampton, United Kingdom \\ Zhq.xu@soton.ac.uk
}

\begin{abstract}
Polypropylene laminated paper (PPLP) is an important composite insulation which has been successfully used in oil filled (OF) cable, mass impregnated (MI) cable and high temperature superconductor (HTS) cable. PPLP is an improved insulation based on conventional cellulose Kraft paper. It is made of one PP film sandwiched between two Kraft papers. The space charge distribution in DDB oil impregnated PPLP sample under DC electric field has been investigated by the pulsed electroacoustic (PEA) technique in this paper. The electric field distribution and distortion have been discussed based on the results obtained from one, two and three layers of PPLP sample. The results show that space charges start to accumulate at the interfaces between paper and PP with time. As a result, the electric field created by the accumulated space charge within the PP film has been enhanced while the electric field in paper reduced. The more the charge accumulated, the higher the electric field distortion in the PP film. The charge distribution and electric field distortion present repeatable pattern in multilayered PPLP sample.
\end{abstract} PEA

Keywords-PPLP; space charge; polypropylene; Kraft paper;

\section{INTRODUCTION}

PPLP (polypropylene laminated paper) is a type of insulation used as an improvement of Kraft paper with excellent performance and high reliability. PPLP insulation has been used in different type of underground transmission cables such as oil-filled (OF) cable, mass-impregnated (MI) cable and high temperature superconductor cable (HTS) [1-4]. High viscosity oil used in MI cables as the impregnated compound, is environment friendly with low risk of oil leaking into the environment. MI cables have been applied under deep water with long distance without oil changing valve.

Because of the higher resistivity and lower permittivity of PP film than that of Kraft paper, electrical stress will mostly be applied on the PP film. It has been found that the improvement in breakdown strength is proportional to PP ratio in PPLP [5]. However, for HVDC applications, space charge dynamics in the oil impregnated PPLP system should be paid more attention. In polymeric cable, easy formation of space charge is a major issue and charge dynamics in the insulation play a critical role in the efficient and reliable HVDC cable operation. Studies about space charge dynamics in oil impregnated PPLP or in liquid nitrogen have been investigated [6-7]. With its potential use for very high voltage DC cable insulation in perspective, it is important to develop a good understanding of charge dynamics in PPLP insulation, especially at the interfaces.

In this paper space charge characteristics in high viscosity DDB oil impregnated commercial PPLP samples have been investigated. Space charge accumulated at interface area can distort the local electric field especially the field across the PP layer. Multi-layered PPLP samples have been studied and the distortion in electric field and its impact on the electrical performance have been discussed.

\section{EXPERIMENT SETUP}

\section{A. Sample preparation}

PPLP is one of the laminated insulation with two layers of Kraft paper sandwiched with one layer of PP (polypropylene). The sample used in this study is the commercial film with very well adhered between Kraft paper and PP. The diagram of PPLP sample is shown in Fig.1. The total thickness of the film is $\sim 120 \mu \mathrm{m}$. The interface of PP and Kraft paper is the mixture two materials as the cellulose structure of Kraft paper allows PP to be pressed into the paper in the laminating processing. They are generally bounded very well and the individual layer of material cannot be tored apart from the film of PPLP. Consequently, the interface in the PPLP is not neat or strict boundary, but a zone of mixture PP and Kraft paper.

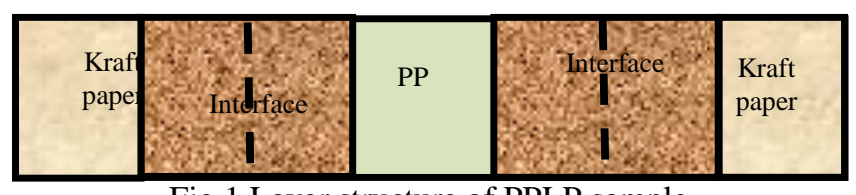

Fig.1 Layer structure of PPLP sample.

The high viscosity DDB oil, used in the real MI cable as impregnant compound, was used in this study. Due to the hygroscopic nature of cellulose, vacuum degassing is necessary. After dried in vacuum oven at $100^{\circ} \mathrm{C}$, the disc PPLP samples were impregnated with degassed DDB oil at $100^{\circ} \mathrm{C}$ under vacuum oven for 2 days. After fully impregnating, the samples were kept in vacuum till the testing.

\section{B. Experimental equipment}

The pulsed electroacoustic (PEA) technique has been considered as the most effective technique for measuring space charge in solid and liquid dielectrics. The PEA system used for

UK Engineering and Physical Sciences Research Council 
the present research has a good spatial resolution and sensitivity, because of a thin PVDF piezoelectric sensor and a narrow width pulse generator selected.

Only positive applied voltage was used in this study. The material of cathode is aluminium and the anode is semiconducting polymer. Since the moisture content is one of the most sensitive factors for oil-paper insulation, all the experiments were carried out at room temperature and under controlled humidity. Before the sample was taken out of the oil container, the impregnated oil moisture was tested and the moisture in all samples was less than 10ppm. The space charge profiles were recorded every $5 \mathrm{mins}$ for $30 \mathrm{mins}$. As the high viscosity cable oil was used, the acoustic velocity of oil, oil Kraft paper and PP are very close. Therefore, the acoustic impedances of different parts of PPLP were similar, making data processing easier.

\section{RESULTS}

\section{A. Charge distribution in single film of PPLP sample.}

Space charge dynamics in the different layer and at interface were studied in this part. In Fig.2, the position of the cathode and anode labelled via black and red solid line. The blue dash lines show the postion of interface zone of Kraft paper and PP layer. The same colour identifier has been consistently used in the rest of figures in this paper.

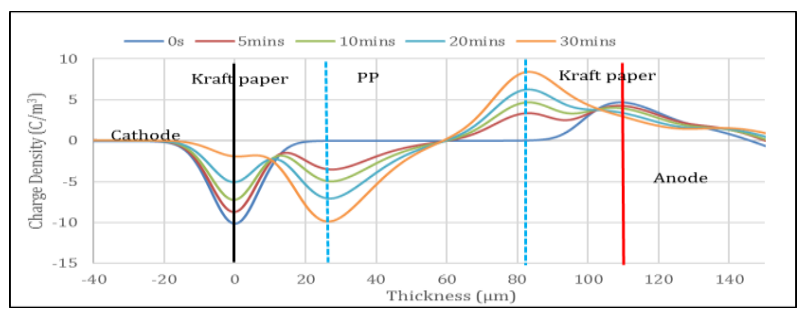

Fig. 1 Space charge distribution in one film of oil-PPLP sample under $+9 \mathrm{kV} / \mathrm{mm}$.

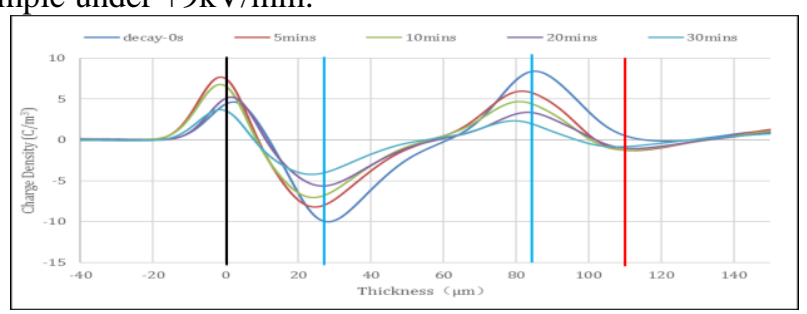

Fig. 3 Space charge decay in one film of oil-PPLP sample under $+9 \mathrm{kV} / \mathrm{mm}$.

In the earliest measurement shown in Fig.2 there is no charge accumulated in the bulk of PPLP sample. The anode peak is bit wider and smaller in magnitude than the cathode peak. This is because of the signal attenuation and scattering of acoustic waves through the PPLP sample. The peaks at both interface zone increase about 3 times during measuring period. At the same time the peak on the electrodes decrease quickly with time. Taking the peak at the cathode as an example, negative charges decrease from $10 \mathrm{C} / \mathrm{m}^{3}$ to $5 \mathrm{C} / \mathrm{m}^{3}$ under the first 5minutes. This is further reduced to about $20 \%$ of the initial charge density after 30 minutes voltage application. The charge density on the anode and in the interface zone next to the anode are tiny bit smaller than the cathode side, but the symmetrical structure shows that changing trend is symmetrical as well.

Fig.3 shows the charge decay curves for one PPLP film after removal of $9 \mathrm{kV} / \mathrm{mm}$ DC supply. This result shows the remaining charges in the dissipation process across the multilayer sample. Charge polarity of the interfacial peaks has the same polarity as the adjacent electrodes. Positive peak found at the cathode is the induced charge caused by the negative charge at the interface. In addition, all peaks decease with time and the decay rate is not very quickly. The magnitude of negative peak at left hand side interface drops down from $10 \mathrm{C} / \mathrm{m}^{3}$ to $4 \mathrm{C} /{ }^{\beta}$ in 30 minutes. Only $60 \%$ charge either dissipates from the electrode or neutralize with the positive charge in PP layer. If the interface area works as traps for charge carriers, the trap depth will be relatively deep. The decay rate is comparable to the charge accumulating rate.

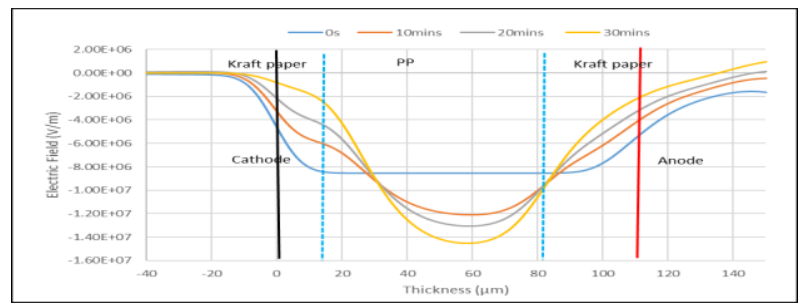

Fig. 4 Electric field distribution in one film of oil-PPLP sample under $+9 \mathrm{kV} / \mathrm{mm}$.

The electric field distribution within the multilayer structure of PPLP insulation can be obtained from Poisson equation based on space charge distribution. As shown in Fig.4, the electric field within both oil impregnated Kraft paper layer, which is marked between electrode and blue line, decease with the period of voltage application. Meanwhile the electric field in the PP layer rises up rapidly after voltage application.

From the Volts-on results, the bipolar charge injection seems to operate and most of injected charges are blocked at the interface zone of Kraft paper and PP mixture. The electric field across the PP layer will be enhanced by the accumulated charge at both interface zones. The more the charge accumulated, the higher the electric field in the PP layer.

Another explanation of the electric distribution in the PPLP film sample is the voltage distribution. If the sample was thought as equivalent circuit, there will be series configuration of different part of materials. And each part could be regarded as the parallel configuration of resistor and capacitor. After the voltage reaches to steady state, the voltage division of different part of the sample is determined by the resistivity of materials. Voltage drops across the PP layer more than that across oil Kraft paper. That will generate higher electric field in the PP as well. Our results are consistent with this analysis qualitatively.

\section{B. Charge distribution in two films of PPLP Sample.}

Fig. 5 shows the space charge distribution in two films of PPLP sample under an applied electric field of $9 \mathrm{kV} / \mathrm{mm}$. The 
negative peak captured from the cathode decreases with time of voltage application, similar to the single film PPLP sample. The negative charges injected from the cathode, migrate to the oil Kraft paper, and are blocked at the left hand side of Kraft paper/PP interface.

From the anode side, marked in red line in Fig.5, the expected positive peak is not observed. One of the reasons is due to the attenuation of acoustic signal. Another reason is the PEA system only measures net charge. The charge at the position of the anode would be the net charge of injected homo charge, the electrode induced charge, and negative ions in the oil Kraft paper moving to there under the applied field.

There are two opposite polarity charge peaks in the middle of two films of PPLP sample. They both increase with time but positive charge peak gets to steady state earlier than negative peak. From the information of sample thickness and acoustic wave velocity, these two peaks are the interface of oil Kraft paper and PP. Because two films of the sample set up in the PEA test cell were two individual impregnated films between PEA electrodes, a thin slice of oil layer would be built up between the films. Our PEA system is hard to get the spatial resolution to separate the charge distribution in that thin layer of oil.

The electrical property of oil insulation paper is highly dependent on the oil condition. The ionization may take place under the external voltage application. As a result, positive charge will move to the cathode part and be blocked at the right PP interface of first PPLP film and negative charge move to the anode part but blocked at the left PP interface of second PPLP film.

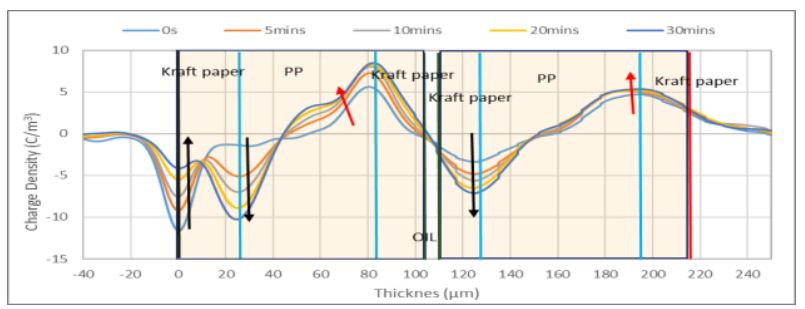

Fig. 5 Space charge distribution of PPLP sample under $9 \mathrm{kV} / \mathrm{mm}$ applied voltage.

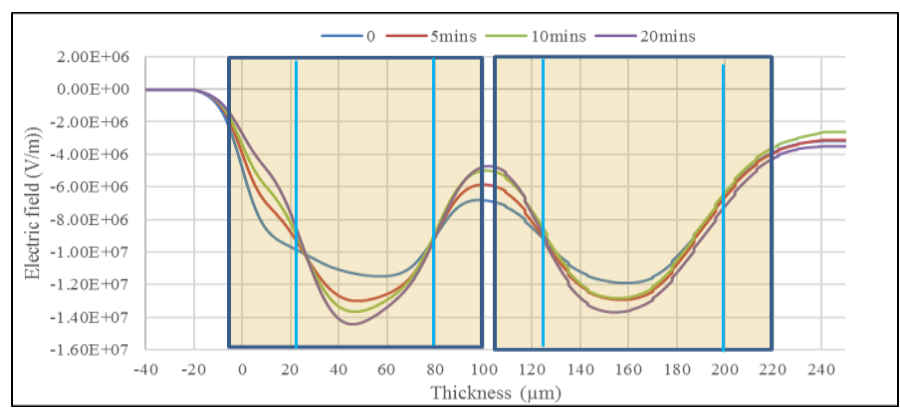

Fig. 6 Electric field distribution of two films of PPLP sample under $9 \mathrm{kV} / \mathrm{mm}$.

Fig.6 shows the electric field distribution calculated by Poisson equation based on the charge accumulated. In each individual layer, a similar pattern has been obtained as in single film sample of section A, shown in Fig.4. The electric field is significant enhanced across the PP film. And the lowest part was found in the oil layer in between two PPLP film. The field across the second film of PPLP next to the anode is a bit smaller than that in first film next to the cathode. This can be attributed to the signal attenuation, which is far away from the acoustic sensor.

\section{Charge distribution in three films of PPLP sample.}

Considering the multi-layered structure of real PPLP insulated cable, three films of PPLP samples were tested under same external electric field. Fig. 7 shows the results of three films of PPLP under $9 \mathrm{kV} / \mathrm{mm}$. Again, the peak at the cathode decrease and peak at left interface of oil Kraft paper with PP increases with the voltage application time. Similar pairs of positive/negative charges are found among the next two films. From both sides of PP layers in each film, samilar distribution patterns are found with negative (left hand side) and positive (right hand side) charge accumulated. As expected, the charge density in the area close to the anode is smaller too.

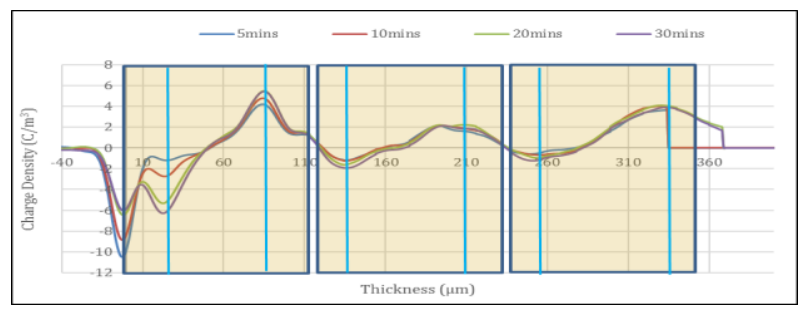

Fig. 7 Space charge distribution of 3 PLPP films of sample under $1 \mathrm{kV}$ applied voltage.

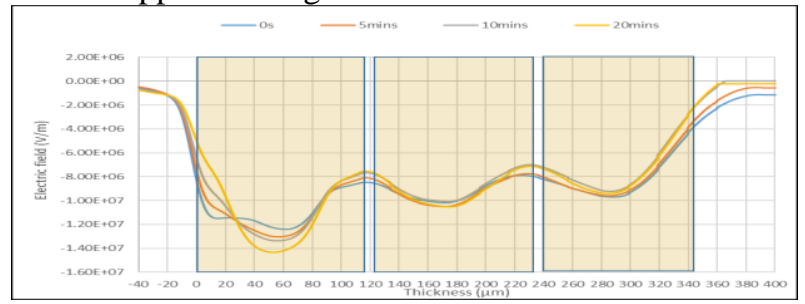

Fig. 8 Electric field distribution of PLPP sample under $1 \mathrm{kV}$ applied voltage.

The electric field distribution across three films PPLP, shown in Fig. 8 is similar with that in the two films. The electric field across the PP layer enhanced because of the accumulated space charge on both sides of PP layer. The lowest parts are located in the oil gaps in between the two films.

\section{DISCUSSTION}

\section{A. Factors that affect space charge distribution in PPLP.}

PPLP is a certainly special insulation, which is nonhomogenized materials in terms of the sandwich structure. When the interfacial space charge in different materials is discussed, the Maxwell-Wagner theory is highly bracketed that the discontinuity of permittivity and conductivity ratio can induce charge at the interface. Firstly, the permittivity and conductivity of different layer of PPLP sample are different. 
However, it is not same case as the oil and oil insulation paper used in transformer. In the transformer, the permittivity of insulation paper is bigger than mineral oil but the conductivity is smaller [8]. However, it is different in oil impregnated PPLP, permittivity of PP is smaller than that of oil Kraft paper and conductivity is smaller as well. Polarization has some effect on the charge distribution but the effect is small. Secondly, the commercial PPLP film, laminated under high temperature and pressure, make the melting PP deeply squeezed into cellulose of insulation paper, and resulting a thinner pure PP layer in the middle of film, as shown in Fig.1. After impregnated with high viscosity insulation oil, the structure will be the symmetric from the inner to the outer as layer of PP, mixture of PP and Kraft paper, then oil Kraft paper. Because of the relative permittivity of PP (2.2) and relative permittivity of DDB oil $(2.3 \pm 0.2)$ are very close and the conductivity of oil Kraft paper and PP are small under $9 \mathrm{kV} / \mathrm{mm}$ electric field. Therefore, the interfacial space charge because of the Maxwell Wagner polarization in the commercial oil impregnated PPLP sample would be small.

Ionization in the oil Kraft paper is another factor that affects the accumulation of space charge. The positive and negative ions would move under the external applied voltage, to the opposite direction. The moisture that oil or Kraft paper absorbed may be ionized and contribute to the charge formed in the test sample. The moisture can affect the conductivity and charge building up speed in the oil Kraft paper as well. Therefore, the sample condition and ambient temperature and relative humidity need to keep consistent. The moisture of impregnated oil was double-checked to be less than 10ppm. However, the PEA system is located in an open area, not in vacuum, which will give small influence of charge movement in the sample. To ensure the influence being relatively kept at the same level, our results presented in this paper were sampling in same day.

Both injected charges and ions will migrate under the influence of the electric field. The physical interface will block or slow down the space charge regardless of ionization or injection. The interface will work as the trapping center for charge carries. The decay results presented in this paper show the slow dissipation process. This indicates the interface area behaves as deep traps for charge carriers.

\section{B. Electric field distortion}

The accumulation of space charge in the insulation sample would affect the electric field distribution. The experimental results show the opposite polarity charges accumulate on both side of PP. The electric field created by the accumulated space charge across the PP film, has the same direction as the applied electric field. Electric field across PP film is enhanced, on the contrary, that across the oil Kraft paper is reduced with time. This is the advantage and reasonable distribution for MI cable with PPLP Insulation working at high voltage level. The breakdown of PP is much higher than that of oil Kraft paper [5]. Higher distorted electric field division on PP layer will give the reasonable representation. At the same time, the higher electric field will force the accumulated charge on both side of PP more chance to inject into PP layer. This was believed as one of the reasons of the interface charge decay slowly when removal the applied voltage. Some of the charge already injected into the PP layer and decay slowly. This also gives the possible risk after the reversing the polarity of the applied voltage. The electric field across the oil Kraft paper would be enhanced after the polarity reversal.

\section{CONLusions}

Space charge distribution and electric field distortion in different layers of laminated dielectric (PPLP) have been investigated under an applied electrical field of $9 \mathrm{kV} / \mathrm{mm}$ in this paper. The following conclusions could be summarized base on the results and discussion.

Interfaces in PPLP insulation structure can block and trap the charges migrating from the electrodes or ions generated within the sample under the external stress. Homo charge injection from both electrodes to the oil Kraft paper layer can be observed. The charge dynamics in the pure PP layer are not very clear to be recognized. Most of charges captured by the PEA are mainly accumulated at Kraft paper/PP interface under $9 \mathrm{kV} / \mathrm{mm}$. The electric field distribution based on the formation of charge show the electric field across the PP layer is significantly enhanced. The more the charge accumulated, the more the electric field distorted. Multi films of PPLP present the repeatable charge distribution pattern. The deeply trapped charges at the interfaces may potentially be harmful when the voltage polarity reversal takes place. It is necessary to have further investigation on the interfacial space charge and electric field behavior for its reliable operation in HVDC cable applications.

\section{ACKNOWLEDGMENT}

The authors are grateful to the financial support received from UK Engineering and Physical Sciences Research Council (EP/L021560/1 - Towards Enhanced HVDC Cable Systems).

\section{REFERENCES}

[1] T. Sugata and R. Hata "Development of $500 \mathrm{kV}$ DC PPLP-Insulated Oil-Filled Submarine Cable", CIGRE SC-21 Paris Meeting, 1996

[2] H. Kubo, N. Noda, I. Nishino, R. Hata, R. T. Miyazaki, "Development of $275 \mathrm{kV}$ Oil-Filled Cable Insulated with Polypropylene Laminated Paper (PPLP), ” Power Engineering Review, IEEE, Vol: PER-2, 1982

[3] R. Hata, "Solid DC submarine cable insulated with polypropylene laminated paper (PPLP)," SEI Technical Review, p. 3-13, 2006.

[4] W. J. Kim, H. J. Kim, J. W. Cho and S. H. Kim, "The Basic Properties of PPLP for HTS DC Cable," Physics Procedia, Vol.45, 2013, pp.293296.

[5] T.Worzyk, M. Bergkvist, "Breakdown voltage Polypropylene Laminated Paper in Plain sample and Full Scale Cable," Conference of Electrical Insulation and Dielectric Phenomena, 1997, pp.329-333.

[6] T. Maeno and K. Fukunaga, "Transient phenomena of space charge ditribtutions in polypropylene laminated paper," International Conference on Conduction and Breakdown in Solid Dielectrics, 1998, pp.43-46.

[7] T. Nakagawa, "Mearsurement of Space Charge Accumulation in PPLP," International Conference on Dielectric Liquids, 1999, pp.533-536.

[8] K.Wu, Q. Zhu, H. Wang,X.Wang and S. Li , "Space Charge Behavior in the Sample with Two Layers of Oil-immersed-paper and Oil," IEEE Transactions on Dielectrics and Electrical Insulation, Vol.21,2014. pp1857-1865. 